\title{
Pointwise Versus Uniform Robustness of Some Large- sample Tests and Confidence Intervals
}

\author{
E. L. LEHMANN \\ University of California, Berkeley \\ WEI-YIN LOH \\ University of Wisconsin, Madison
}

\begin{abstract}
The true level $\boldsymbol{\alpha}_{n}(F)$ of a test at nominal level $\alpha$ and sample size $\boldsymbol{n}$ is said to be point wise robust if $\alpha_{n}(F) \rightarrow a$ as $n \rightarrow \infty$ for each distribution $F$. If $F$ is unknown and only assumed to belong to the class $F$ of distributions, one may wish to consider the level to be defined by $\alpha_{n}=\sup _{\mathscr{F}} a_{n}(F)$. The test is said to be uniformly robust over $F$ if $\alpha_{n} \rightarrow \alpha$ as $n \rightarrow \infty$. Corresponding definitions apply to the coverage probabilities of confidence intervals.

The purpose of this paper is to illustrate the fact that it is typical for many large-sample tests to be pointwise robust but not uniformly robust, even for seemingly narrow classes $F$. Two cases, the one-sample $t$-test and confidence intervals for the binomial proportion $p$, are treated in detail. It is shown that the $t$-test is not uniformly robust if $F$ consists of distributions within a Kolmogorov neighbourhood of an arbitrary distribution or those with densities supported in a finite interval. The test is uniformly robust if $F$ is a set of distributions with uniformly bounded standardized moments. In the binomial case it is shown that the standard confidence intervals constructed using the delta method are not uniformly robust if $\mathscr{F}$ consists of all binomial distributions with $0<p<1$.
\end{abstract}

Key words: Kolmogorov distance, significance level

\section{Introduction}

Let $\mathbf{X}_{n}=\left(X_{1}, \ldots, X_{n}\right)$ be i.i.d. according to $N\left(\theta, \sigma^{2}\right)$, the normal distribution with mean $\theta$ and variance $\sigma^{2}$, and let $\Phi(\cdot)$ be the standard normal c.d.f. The level- $\alpha$ t-test of $\mathscr{H}: \theta=0$ against $\theta>0$ rejects when

$$
t_{n}=(n)^{1 / 2} s_{n}^{-1} \bar{X} \geqslant c_{n}
$$

where $\bar{X}=n^{-1} \sum_{i=1}^{n} X_{i}$ and $s_{n}^{2}=(n-1)^{-1} \sum_{i=1}^{n}\left(X_{i}-\bar{X}\right)^{2}$, and where

$$
c_{n} \rightarrow z_{\alpha} \text { as } n \rightarrow \infty
$$

with $z_{\alpha}$ defined by $1-\Phi\left(z_{\alpha}\right)=\alpha$.

Suppose now that the assumption of normality is not justified (as it never is), and that instead $\mathbf{X}_{n}$ is a sample from some other distribution $F$ with mean $\mu_{F}=0$. Let $\alpha_{n}(F)$ denote the probability of rejecting the hypothesis that $\mu_{F}$ is 0 . Then a standard result (see, e.g. Lehmann, 1986) states that

$$
\alpha_{n}(F) \rightarrow \alpha \text { as } n \rightarrow \infty
$$

for any $F$ with finite variance. This is often described by saying that the level of the $t$-test is robust against non-normality. For any such $F$, the rejection probability under $\mathscr{H}$ will then be close to $\alpha$ if $n$ is sufficiently large. How large $n$ needs to be for the actual level $\alpha_{n}(F)$ to be close to the nominal level $\alpha$ has been much studied in the literature. Some recent references are Cressie (1980), Benjamini (1983), Herrendoerfer, Rasch \& Feige (1983), and Herrendoerfer $\&$ Feige (1985). A universal answer could be given if the convergence of $\alpha_{n}(F)$ to $\alpha$ is uniform in $F$. Otherwise, the answer must depend on $F$. 
There is another way of looking at the robustness of the $t$-test. If $F$ is unknown, one may wish to consider the $t$-test as a test of the hypothesis

$$
W^{\prime} \text { : the mean } \mu_{F} \text { of the unknown distribution } F \text { is } 0
$$

against the alternatives $\mathscr{K}^{\prime}$ that $\mu_{F}$ is positive. As a test of $\mathscr{H}^{\prime}$ the level of the $t$-test is then

$$
\alpha_{n}=\sup _{\not} \alpha_{n}(F)
$$

where $F$ is the class of all distributions $F$ with mean zero and finite variance. Robustness of the $t$-test in this setting would require

$$
\alpha_{n} \rightarrow \alpha \text { as } n \rightarrow \infty .
$$

If (4) holds, we shall say that the test is uniformly robust over $\mathbb{F}$. Clearly (4) implies that the convergence of $\alpha_{n}(F)$ to $\alpha$ is uniform over $\mathscr{F}$.

Unfortunately, as was shown in Bahadur \& Savage (1956) in a more general context, (4) does not hold. On the contrary, for the $t$-test (1), one has by Bahadur \& Savage (1956) that

$$
\sup _{\not{Z}} \alpha_{n}(F)=1
$$

for every value of $\alpha$. (The same result then follows trivially for the two-sided $t$-test. See Gleser \& Hwang, 1987, for a similar result in an errors-in-variables model.)

In the light of (5) the question about the sample size required for the level $\alpha_{n}(F)$ of the $t$-test to be reasonably close to $\alpha$, must be approached somewhat differently. Given a small $\varepsilon>0$, no sample size $n_{0}$ can insure that $\left|\alpha_{n}(F)-\alpha\right|<\varepsilon$ for all $n>n_{0}$. However, one may hope that such an $n_{0}$ will exist if $F$ is restricted to a suitable subset $\mathscr{F}_{0}$ of $\mathscr{F}$ that includes all distributions likely to obtain in the given situation. We shall examine this possibility for several definitions of $\mathscr{F}_{0}$ in the next section.

Let us briefly mention two other approaches to the problem.

1. Although the $t$-test does not satisfy (4), there might exist another test that does. Suppose $\varphi$ is any test that in the original normal model has power $\beta>\alpha$ against some normal alternative $N\left(\theta, \sigma^{2}\right)$ with $\theta>0$. Then given any $\varepsilon>0$ it follows from Bahadur \& Savage (1956) that there exists a distribution $F \in \mathscr{F}$ with mean zero and $\alpha_{n}(F)>\beta-\varepsilon$ and hence such a test will satisfy $\alpha_{n} \geqslant \beta$. In particular, if its power tends to 1 as $\theta \rightarrow \infty$, then the test will satisfy (5).

2. Without much consideration, we generalized the original hypothesis $\mathscr{H}$ that the mean of the assumed normal distribution is zero to the hypothesis $\mathscr{C}^{\prime}$ defined by (3). However, under the assumption of normality, $\theta$ is not only the mean of the $X$ 's; it is also the median, the symmetrically trimmed mean, and so on, since all these measures of location coincide for a symmetric distribution.

If such an alternative generalization :s adopted, the results can be different. For example, let $v$ be the median of $F$, so that $F(v)=1 / 2$. Then the sign test can be used to test $\mathscr{H}^{\prime \prime}: v=0$ against the alternatives that $v>0$. If $S_{n}$ denotes the number of positive $X$ 's, then under $\mathscr{C}^{\prime \prime}, S_{n}$ has the binomial distribution corresponding to $n$ trials and success probability $p=1 / 2$, and its level is therefore identically equal to $\alpha$, independent of $F$, and hence trivially satisfies $\alpha_{n}=\alpha$.

Whether the mean, median, or some other measure of location is the most appropriate extension of $\theta$ (in the normal case) when arbitrary distributions $F$ are considered possible depends on the context of the particular problem. For a discussion, see for example Lehmann (1975, pages 84-85). 
The discussion of robustness of the significance level of the one- and two-sided $t$-test translates in the obvious way to the confidence coefficients of the corresponding confidence bounds and intervals. Furthermore, the discussion extends easily to other tests concerning normal means, for example, the linear models $F$-test.

The situation is different for the corresponding tests or confidence sets for a normal variance, the ratio of two variances, or the correlation coefficient. These procedures are not even robust, i.e. don't even satisfy (2) (cf. Miller, 1986). They can be made robust, essentially through studentization. The resulting robust procedures behave similarly to the $t$-test and are not uniformly robust.

\section{Uniform and non-uniform robustness of the $t$-test}

In this section, we shall consider some families $\mathscr{F}_{0}$ of "reasonable" distributions and for each ask whether it entails

$$
\sup _{\not_{0}} \alpha_{n}(F) \rightarrow \alpha \quad \text { as } \quad n \rightarrow \infty .
$$

\subsection{Kolmogorov-Smirnov neighbourhoods}

It would seem natural to hope for the validity of (6) when $\mathscr{F}_{0}$ is the family of all distributions not differing much from the family of normal distributions with mean zero. One well-known measure of closeness between two distributions is the Kolmogorov-Smirnov distance. It turns out that not only the $t$-test, but any non-trivial unbiased test of the hypothesis that $\mu_{F}=0$ is not uniformly robust over Kolmogorov-Smirnov neighbourhoods.

To see this, let $\Delta>0$ and let $F_{0}$ be a fixed distribution with mean zero and bounded density function $f_{0}(x)$, and let $\mathscr{F}_{01}$ be the family of all distributions for which

$$
\left|F(x)-F_{0}(x / \sigma)\right|<\Delta \text { for all } x \text { and some } \sigma>0 .
$$

Let $U(a, h)$ denote the uniform distribution on the interval $(a-h, a+h)$ and let $\omega=\sup _{x} f_{0}(x)$. Consider the mixture c.d.f

$$
F(x)=(1-\lambda) F_{0}\left(x-\xi_{2}\right)+\lambda G_{\xi_{1}, \lambda}(x),
$$

where

$$
\begin{aligned}
& 0<\lambda<1, \quad 0<\xi_{2}<\omega^{-1} \Delta, \\
& \xi_{1}=-\lambda^{-1}(1-\lambda) \xi_{2},
\end{aligned}
$$

and $G_{\xi_{1}, \lambda}(x)$ is the cdf of the $U\left(\xi_{1}, \lambda\right)$ distribution.

Because for this $F$,

$$
\begin{aligned}
\left|F(x)-F_{0}(x)\right| & \leqslant\left|(1-\lambda) F_{0}(x)-F_{0}(x)\right|+(1-\lambda)\left|F_{0}(x)-F_{0}\left(x-\xi_{2}\right)\right|+\lambda G_{\xi_{1}, \lambda}(x) \\
& \leqslant 2 \lambda+(1-\lambda) \omega \xi_{2}
\end{aligned}
$$

it follows from the above conditions on $\xi_{1}$ and $\xi_{2}$ that (8) belongs to $\not{F}_{01}$ for all $\lambda$ sufficiently small.

Let $\varphi$ be any test of the hypothesis $\mathscr{H}^{\prime}: \mu_{F}=0$ and let $\beta_{n}\left(\omega^{-1} \Delta ; \varphi\right)$ be its power against the alternative $F_{0}\left(x-\omega^{-1} \Delta\right)$. Suppose $\mathscr{S}$ denotes the rejection region of the $\varphi$-test and let $\alpha_{n}^{(\varphi)}(F)$ be the probability that $\varphi$ rejects $\mathscr{H}^{\prime}$ based on a sample of size $n$ from $F$. Then

$$
\alpha_{n}^{(\varphi)}(F) \geqslant(1-\lambda)^{n} \operatorname{Pr}_{\xi_{2}}\left\{\left(X_{1}, \ldots, X_{n}\right) \in \mathscr{S}\right\}
$$


where $\operatorname{Pr}_{\xi_{2}}$ indicates that the probability is calculated under $F_{0}\left(x-\xi_{2}\right)$. It follows by letting $\lambda \rightarrow 0$ and $\xi_{2} \rightarrow \omega^{-1} \Delta$ that

$$
\sup _{\widetilde{Z}_{01}} \alpha_{n}^{(\varphi)}(F) \geqslant \beta_{n}\left(\omega^{-1} \Delta ; \varphi\right) \quad \forall F_{0}, \varphi, n, \text { and } \quad \Delta>0 .
$$

We therefore have proved the following result.

\section{Theorem 1}

Let $F_{0}$ be any distribution with mean zero and bounded density function $f_{0}(x)$ and let $\omega=\sup _{x} f_{0}(x)$. Given $\Delta>0$, let $\mathscr{F}_{01}$ be the Kolmogorov-Smirnov neighbourhood (7) around the scale-family induced by $F_{0}$. Then (9) obtains for any test $\varphi$ of the hypothesis that the mean is zero.

Specializing $\varphi$ to be the $t$-test and $F_{0}$ to be the standard normal distribution yields the following corollary.

\section{Corollary 1}

Let $F_{0}$ be the standard normal distribution. Then

$$
\sup _{\mathcal{F}_{01}} \alpha_{n}(F) \rightarrow 1 \quad \text { as } \quad n \rightarrow \infty, \quad \forall 0<\alpha<1 \text {. }
$$

\subsection{Uniformly bounded support}

Since $\xi_{1} \rightarrow-\infty$ as $\lambda \rightarrow 0$ in the proof of the theorem, it might be thought that the cause of the difficulty with the family defined in (7) is that it is possible to cause a perturbation arbitrarily far out in the tail of the distribution. This may lead to the conjecture that the difficulty will disappear if $\mathscr{F}_{0}$ is restricted to contain only distributions with uniformly bounded support.

\section{Theorem 2}

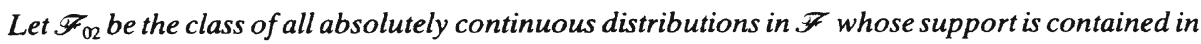
a bounded interval. Then for every $0<\alpha \leqslant 1$,

$$
\sup _{\mathbb{Z}_{02}} \alpha_{n}(F)=1 \text { for all sufficiently large } n \text {. }
$$

Proof. Without loss of generality, assume that the support of each $F \in \mathbb{F}_{02}$ is contained in the interval $(-1,1)$. Consider the distribution

$$
F=(1-\lambda) U(-\lambda, \lambda / 2)+\lambda U(1-\lambda, \lambda / 2) .
$$

It is easily verified that $F \in \mathscr{F}_{02}$ for $0<\lambda<2 / 3$. Let $\mathscr{C}$ denote the event that the entire sample $X_{1}, \ldots, X_{n}$ comes from the first component $U(-\lambda, \lambda / 2)$. When $\mathscr{A}$ occurs, the minimum value of $|\bar{X}|$ is $\lambda / 2$ and the maximum value of $s_{n}$ is $\lambda / 2$ if $n$ is odd, and $(\lambda / 2) \sqrt{n(n-1)^{-1}}$ if $n$ is even. This yields $\left|t_{n}\right| \geqslant \sqrt{n-1}$. Therefore for all large $n$ (independent of $\lambda$ )

$$
\alpha_{n}(F) \geqslant \operatorname{Pr}(., t)=(1-\lambda)^{n} .
$$

Letting $\lambda \rightarrow 0$ in (11) yields (10).

\subsection{Uniformly bounded standardized moments}

Let $\sigma_{F}$ denote the standard deviation of $F$ and let $\chi_{r}(F)=E_{F}\left|X-\mu_{F}\right|^{r} / \sigma_{F}^{r}$ denote the $r$ th standardized absolute moment about the mean of $F$. Let $\mathscr{F}_{03}$ be a class of absolutely con- 
tinuous $F \in \mathscr{F}$ with $\varkappa_{s}(F)$ uniformly bounded for some $s=2+\delta, \delta>0$. It turns out that

$$
\sup _{\exists_{03}} \alpha_{n}(F) \rightarrow \alpha \text { as } n \rightarrow \infty \text {. }
$$

Result (12) is easily proved using a Berry-Esséen theorem for the $t$-statistic in Chung (1946) if we assume that $\varkappa_{8}(F)$ is uniformly bounded. A proof of the more general result was communicated to us by $T$. Severini. We give a sketch of it via the following lemmas.

\section{Lemma 1}

Let $Y_{1}, Y_{2}, \ldots$, be a sequence of i.i.d. random variables with means 0 and distribution $F \in \mathscr{C}$, where $\mathscr{C}$ satisfies

$$
\sup _{\mathscr{E}} E\left|Y_{1}\right|^{1+\delta}<\infty
$$

for some $\delta>0$. Then for every $\varepsilon>0$,

$$
\lim _{n \rightarrow \infty} \sup _{\mathscr{\varepsilon}} \operatorname{Pr}\left(\left|n^{-1} \sum_{i=1}^{n} Y_{i}\right|>\varepsilon\right)=0
$$

Proof. The result is a consequence of Chebychev's inequality if $\delta=1$. For the general case, define $Z_{j}=Y_{j}$ if $\left|Y_{j}\right| \leqslant n$, and 0 otherwise, for $j=1, \ldots, n$. Then $\operatorname{Var}\left(Z_{j}\right) \leqslant n^{-1 \delta} M$ and $E\left|Y_{j}-Z_{j}\right| \leqslant n^{-\delta} M$, where $M=\sup _{\mathscr{E}} E\left|Y_{1}\right|^{1+\delta}$. Hence for any $\varepsilon>0$,

$$
\operatorname{Pr}\left\{\left|n^{-1} \sum_{j=1}^{n}\left(Y_{j}-Z_{j}\right)\right|>\varepsilon\right\} \leqslant n^{-\delta} \varepsilon^{-1} M
$$

and

$$
\operatorname{Pr}\left(\left|n^{-1} \sum_{j=1}^{n} Z_{j}\right|>\varepsilon\right) \leqslant n^{-\delta} \varepsilon^{-2} M
$$

with both inequalities holding uniformly over $\mathscr{C}$. This implies that

$$
\sup _{\mathscr{G}} \operatorname{Pr}\left(\left|n^{-1} \sum_{j=1}^{n} Y_{j}\right|>2 \varepsilon\right) \leqslant n^{-\delta} M \varepsilon^{-2}(1+\varepsilon)
$$

for all $n$ and every $\varepsilon>0$, and the lemma follows.

\section{Lemma 2}

Let $Y_{1}, Y_{2}, \ldots$ be a sequence of i.i.d. random variables with mean $\eta$, variance $\tau^{2}$, and distribution $F \in \mathscr{D}$, where $\mathscr{D}$ satisfies

$$
\sup _{\mathscr{Z}} E\left|\tau^{-1}\left(Y_{1}-\eta\right)\right|^{2+\delta}<\infty
$$

for some $\delta>0$. Let $\bar{Y}_{n}=n^{-1} \sum_{j=1}^{n} Y_{j}$ and $\hat{\tau}_{n}^{2}=n^{-1} \sum_{j=1}^{n}\left(Y_{j}-\bar{Y}_{n}\right)^{2}$. Then for every $\varepsilon>0$,

$$
\sup _{\mathscr{Z}} \operatorname{Pr}\left(\tau^{-1}\left|\hat{\tau}_{n}-\tau\right|>\varepsilon\right) \rightarrow 0 \quad \text { as } n \rightarrow \infty \text {. }
$$

Proof. Let $Z_{j}=\tau^{-1}\left(Y_{j}-\eta\right)$. Then $E\left(Z_{j}^{2}-1\right)=0$ and

$$
\sup _{\mathscr{I}} E\left|Z_{j}^{2}-1\right|^{1+\delta / 2} \leqslant 2^{\delta / 2}\left(\sup _{\mathscr{D}} E\left|Z_{j}\right|^{2+\delta}+1\right)<\infty \text {. }
$$


It follows from lemma 1 that, for every $\varepsilon>0$,

$$
\sup _{\mathscr{Q}} \operatorname{Pr}\left(\left|n^{-1} \sum_{=1}^{n} Z_{j}^{2}-1\right|>\varepsilon\right) \rightarrow 0
$$

as $n \rightarrow \infty$. Similarly, because $E Z_{j}=0$ and $\sup _{\mathscr{Q}} E\left|Z_{j}\right|^{1+\delta}<\infty$, lemma 1 implies that

$$
\sup _{\mathscr{Z}} \operatorname{Pr}\left(\left|n^{-1} \sum_{j=1}^{n} Z_{j}\right|>\varepsilon\right) \rightarrow 0
$$

as $n \rightarrow \infty$ for every $\varepsilon>0$. Finally, because

$$
\tau^{-2} \hat{\tau}_{n}^{2}=n^{-1} \sum_{j=1}^{n} Z_{j}^{2}-\left(n^{-1} \sum_{j=1}^{n} Z_{j}\right)^{2},
$$

we have

$$
\sup _{\mathscr{P}} \operatorname{Pr}\left(\left|\tau^{-2} \hat{\tau}_{n}^{2}-1\right|>\varepsilon\right) \rightarrow 0
$$

for every $\varepsilon>0$.

Proof of (12). Let $W_{n}=n^{-1 / 2}\left(\sum_{i=1}^{n} X_{i}-n \mu_{F}\right), G_{n, F}(x)=\operatorname{Pr}_{F}\left\{\sigma_{F}^{-1} W_{n} \leqslant x\right\}$, and $\hat{G}_{n, F}(x)=$ $\operatorname{Pr}_{F}\left\{s_{n}^{-1} W_{n} \leqslant x\right\}$. If $\varkappa_{2+\delta}(F)$ is uniformly bounded for some $\delta>0$, the Berry-Esséen theorem for sums of independent random variables (see, e.g. Prakasa Rao, 1987, page 25) implies that

$$
\sup _{\mathcal{P}_{03}} \sup _{x}\left|G_{n, F}(x)-\Phi(x)\right| \rightarrow 0 \quad \text { as } n \rightarrow \infty .
$$

It therefore follows that, for every $\varepsilon>0$,

$$
\begin{aligned}
\left|\hat{G}_{n, F}(x)-\Phi(x)\right| \leqslant & \left|G_{n, F}(x)-\Phi(x)\right|+\left|\hat{G}_{n, F}(x)-G_{n, F}(x)\right| \\
\leqslant & \left|G_{n, F}(x)-\Phi(x)\right|+\operatorname{Pr}\left(\sigma_{F}^{-1}\left|s_{n}-\sigma_{F}\right|>\varepsilon\right) \\
& +\left|\operatorname{Pr}\left(\left\{s_{n}^{-1} W_{n} \leqslant x\right\} \cap\left\{\sigma_{F}^{-1}\left|s_{n}-\sigma_{F}\right| \leqslant \varepsilon\right\}\right)-G_{n, F}(x)\right| \\
\leqslant & \left|G_{n, F}\{x(1+\varepsilon)\}-\Phi\{x(1+\varepsilon)\}\right|+2\left|G_{n, F}(x)-\Phi(x)\right| \\
& +\left|G_{n, F}\{x(1-\varepsilon)\}-\Phi\{x(1-\varepsilon)\}\right|+2 \operatorname{Pr}\left(\sigma_{F}^{-1}\left|s_{n}-\sigma_{F}\right|>\varepsilon\right) \\
& +|\Phi\{x(1+\varepsilon)\}-\Phi(x)|+|\Phi\{x(1-\varepsilon)\}-\Phi(x)| \\
\rightarrow & 0
\end{aligned}
$$

uniformly over $x$ and $\mathscr{F}_{03}$ as $n \rightarrow \infty$ and $\varepsilon \rightarrow 0$.

Following are some examples of $\mathscr{F}_{03}$ that are determined by the shapes of the distributions. Throughout, $S_{F}$ will denote the support of $F$.

\subsubsection{Symmetric distributions defined by shape}

Let $F_{0}$ be a fixed, symmetric, absolutely continuous distribution with mean zero and finite $(2+\delta)$ th moment, for some $\delta>0$. Let $\bar{F}_{04}$ be the family of absolutely continuous symmetric distributions with zero mean that precede $F_{0}$ according to the $s$-ordering $<_{s}$ of van Zwet (1964):

$F{ }_{s} G \Leftrightarrow G^{-1} F(x)$ is concave-convex about the point of symmetry of $F$. 
This is a partial ordering on the set of symmetric distributions according to shape. Intuitively, $F<_{s} G$ if $F$ is less peaked as well as less heavily-tailed than $G$. Van Zwet (1964, theorem 2.3.2) showed that $\varkappa_{r}(F)$ is isotonic with respect to the $s$-ordering for all $r \geqslant 2$, i.e.

$$
F<_{s} G \Rightarrow \varkappa_{r}(F) \leqslant \varkappa_{r}(G) \forall r \geqslant 2 .
$$

Hence

$$
\sup _{\mathcal{Z}_{04}} \varkappa_{2+\delta}(F) \leqslant \varkappa_{2+\delta}\left(F_{0}\right)
$$

and (6) holds for $\mathscr{F}_{0}=\mathscr{F}_{04}$.

Some examples are:

1. $\mathscr{F}_{04}=\{F: f(x)$ is symmetric and $U$-shaped $\}$, because every symmetric distribution with a $\mathrm{U}$-shaped density $s$-precedes the uniform distribution.

2. Define $\tilde{F}(x)=2 F\left(x+\mu_{F}\right)-1$. We may let $\mathscr{F}_{04}=\{F: F$ is symmetric and $\tilde{F}$ is an increasing failure rate distribution (IFR) $\}$, because if $\tilde{F}$ is IFR, then $F<_{s}$ double exponential.

3. $\mathscr{F}_{04}=\{F: F$ is a symmetric and strongly unimodal distribution $\}$, because the family of symmetric strongly unimodal distributions is a subfamily of the family of $F$ such that $\bar{F}$ is IFR (see Loh, 1984). Recall that a non-degenerate strongly unimodal distribution is one whose density is absolutely continuous and log-concave on its support (Ibragimov, 1956). This family includes the uniform, normal, logistic, and double exponential distributions.

\subsubsection{Neighbourhoods around normal defined by moment conditions}

Given $\delta, \Delta>0$, let $F_{05}$ be the family of distributions in $F$ which are close to the normal scalefamily in the sense that

$$
\left|x_{2+\delta}(F)-x_{2+\delta}(\Phi)\right|<\Delta \text {. }
$$

Then $\varkappa_{2+\delta}(F)$ is uniformly bounded and (6) holds for $\mathscr{F}_{0}=\mathscr{F}_{05}$.

\subsubsection{Neighbourhoods around non-normal $F$}

It is clear that the result of section 2.3.2 remains valid if in (13) the normal c.d.f. $\Phi$ is replaced by any non-degenerate distribution $F_{1}$ with finite $(2+\delta)$ th moment for some $\delta>0$. This fact may be described by saying that the $t$-test is locally uniformly robust for the neighbourhoods defined by

$$
\left|x_{2+\delta}(F)-x_{2+\delta}\left(F_{1}\right)\right|<\Delta
$$

for some $\delta>0$.

\section{Intervals for binomial $p$}

Let $Y$ have a binomial distribution $B(p, n)$ with parameters $p$ and $n$. Exact confidence intervals for $p$ exist and can be found in published tables. We discuss in this section some problems concerning large-sample intervals for $p$ from the point of view of sections 1 and 2 . We will say that a nominal $100(1-\alpha) \%$ confidence interval $I_{n}$ for $p$ constructed from $n$ observations is uniformly robust over a class of distributions $\mathscr{F}$ if

$$
\inf _{\nexists} \operatorname{Pr}_{p}\left(p \in I_{n}\right) \rightarrow 1-\alpha \text { as } n \rightarrow \infty \text {. }
$$




\subsection{Normal-approximation interval}

Consider the standard $100(1-\alpha) \%$ "normal-approximation" interval $I_{n}$ with endpoints

$$
\hat{p} \pm z_{a / 2} n^{-1 / 2} \sqrt{\hat{p}(1-\hat{p})},
$$

where $\hat{p}=Y / n$. This interval reduces to the singleton $\{0\}$ or the empty set (depending on whether $I_{n}$ is taken to be closed or open) whenever $Y=0$. Suppose that $I_{n}$ is the open interval and let $\mathscr{G}_{0}$ be the set of all distributions $B(p, n)$ with $0<p<1$. The interval is clearly pointwise consistent for each $p$ in $(0,1)$. Because $\operatorname{Pr}_{p}(\hat{p}=0) \rightarrow 1$ as $p \rightarrow 0$, however (see e.g. Blyth \& Still, 1983, p. 114),

$$
\inf _{p \in(0,1)} \operatorname{Pr}_{p}\left(p \in I_{n}\right)=0 \text { for every } n .
$$

Therefore (14) is not satisfied, i.e. $I_{n}$ is not uniformly robust over $\mathscr{G}_{0}$.

Let $0<a<b<1$. It turns out that

$$
\lim _{n \rightarrow \infty} \inf _{p \in(a, b)} \operatorname{Pr}_{p}\left(p \in I_{n}\right)=1-\alpha
$$

and

$$
\lim _{n \rightarrow \infty} \sup _{p \in(a, b)} \operatorname{Pr}_{p}\left(p \in I_{n}\right)=1-\alpha .
$$

That is, $I_{n}$ is locally robust.

Proof of (16) and (17). Let $q=1-p, c=z_{\alpha / 2}$ and $\gamma_{n}=\operatorname{Pr}_{p}\left(p \in I_{n}\right)$. Then for large enough $n$,

$$
\begin{aligned}
\gamma_{n}= & \operatorname{Pr}_{p}\left\{\hat{p}-p \in \frac{(1-2 p) c^{2} n^{-1} \pm c n^{-1 / 2} \sqrt{4 p(1-p)+c^{2} n^{-1}}}{2\left(1+c^{2} n^{-1}\right)}\right\} \\
& \geqslant \operatorname{Pr}_{p}\left\{\frac{(Y-n p)}{\sqrt{n p q}} \in \frac{(1-2 p) c^{2}(n p q)^{-1 / 2} \pm c\left[2-c(n p q)^{-1}\right]}{2\left(1+c^{2} n^{-1}\right)}\right\} \\
& >\operatorname{Pr}_{p}\left\{Z \in \frac{(1-2 p) c^{2}(n p q)^{-1 / 2} \pm c\left[2-c(n p q)^{-1 / 2}\right]}{2\left(1+c^{2} n^{-1}\right)}\right\}-\frac{C \varrho_{n}}{\sigma_{n}^{3} n^{1 / 2}}
\end{aligned}
$$

Here $\sigma_{n}^{2}$ and $\varrho_{n}$ denote the variance and third absolute moment about the mean of $Y$ respectively, $Z$ denotes the $N(0,1)$ random variable, and $C$ is a universal constant. The second inequality follows from the Berry-Esséen theorem (see e.g. Feller, 1966, p. 515). Because $p$ is bounded away from 0 and 1 , the two quantities $(n p q)^{-1 / 2}$ and $\varrho_{n} / \sigma_{n}^{3} n^{1 / 2}$ tend to zero as $n \rightarrow \infty$. Thus

$$
\liminf _{n \rightarrow \infty} \inf _{p \in(a, b)} \operatorname{Pr}_{p}\left(p \in I_{n}\right) \geqslant 1-\alpha .
$$

Similarly,

$$
\gamma_{n}<\operatorname{Pr}_{p}\left\{Z \in \frac{(1-2 p) c^{2}(n p q)^{-1 / 2} \pm c\left[2+c(n p q)^{-1 / 2}\right]}{2\left(1+c^{2} n^{-1}\right)}\right\}+\frac{C \varrho_{n}}{\sigma_{n}^{3} n^{1 / 2}},
$$

which implies that

$$
\underset{n \rightarrow \infty}{\limsup } \sup _{p \in(a, b)} \operatorname{Pr}_{p}\left(p \in I_{n}\right) \leqslant 1-\alpha .
$$


The desired results now follow from (18) and (19).

The proof shows that it is not necessary for $a$ and $b$ to be held fixed as $n$ increases. The same results hold if we allow the neighbourhood to be $\left(a_{n}, b_{n}\right)$, where $a_{n} \rightarrow 0$ and $b_{n} \rightarrow 1$ in such a way that

$$
\sup _{p \in\left(a_{n}, b_{n}\right)}(n p q)^{-1}=o(1), \sup _{p \in\left(a_{n}, b_{n}\right)} \varrho_{n} \sigma_{n}^{-3}=o\left(n^{1 / 2}\right) .
$$

Suppose now that the family $\mathscr{G}_{0}$ is enlarged to include the degenerate binomial distributions corresponding to $p=0$ and $p=1$. Let $\mathscr{G}_{1}$ denote the enlarged family. The interval $I_{n}$ is still pointwise consistent over $\mathscr{G}_{1}$. However, it is no longer locally uniformly robust at $B(0, n)$ and $B(1, n)$. Thus $I_{n}$ is locally uniformly robust at each distribution in $\mathscr{G}_{0}$ but not $\mathscr{G}_{1}$. This shows that local uniform robustness can be rather a fragile property which may be destroyed by slight perturbations of the problem.

\subsection{Arcsine transformation}

It may be thought that the failure of the interval (15) to be uniformly robust over $\mathscr{G}_{0}$ and locally uniformly robust over $\mathscr{G}_{1}$ is because the width of the interval shrinks to zero when $Y=0$ or $n$. The arcsine transformation is sometimes recommended because $\arcsin (\sqrt{\hat{p}})$ is more nearly normally distributed than is $\hat{p}$ and also because the variance of the former is approximately a constant equal to $(4 n)^{-1}$ (see e.g. Johnson \& Kotz, 1969, p. 65). Applying the normal approximation to $\arcsin (\sqrt{\hat{p}})$, we get the following approximate $100(1-\alpha) \%$ confidence interval, $J_{n}$ say, for $p$ :

$$
\sin ^{2}\left\{\arcsin (\sqrt{\hat{p}})-z_{\alpha / 2}\left(2 n^{1 / 2}\right)^{-1}\right\}^{+}<p<\sin ^{2}\left\{\arcsin (\sqrt{\hat{p}})+z_{\alpha / 2}\left(2 n^{1 / 2}\right)^{-1}\right\} .
$$

Note that the width of $J_{n}$ does not vanish when $\hat{p}=0$ or 1 . Unfortunately, $J_{n}$ is also not uniformly robust over $\mathscr{G}_{0}$ when $0<\alpha<1 / 2$ because

$$
\limsup _{n \rightarrow \infty} \inf _{p \in(0,1)} \operatorname{Pr}_{p}\left(p \in J_{n}\right)<1-\alpha, \quad 0<\alpha<1 / 2 .
$$

Proof of (20). When $Y=0, J_{n}$ reduces to the interval $\left(0, u_{n}\right)$, where $u_{n}=\sin ^{2}\left\{z_{a / 2}\left(2 n^{1 / 2}\right)^{-1}\right\}$. Let $p=u_{n}$ and $v_{n}=1-u_{n}$. Then the probability that $J_{n}$ does not contain $p$ is at least $v_{n}^{n}$, which for large $n$ is approximately $\left\{1-z_{\alpha / 2}^{2}(4 n)^{-1}\right\}^{n}$. The last expression converges as $n \rightarrow \infty$ to $\exp \left(-4^{-1} z_{\alpha / 2}^{2}\right)$. It follows that

$$
\underset{n \rightarrow \infty}{\limsup } \inf _{p \in(1,1)} \operatorname{Pr}_{p}\left(p \in J_{n}\right) \leqslant 1-\exp \left(-4^{-1} z_{\alpha / 2}^{2}\right)
$$

Using the inequality (see Abramowitz \& Stegun, 1965, p. 933, formula 26.2.23)

$$
z_{\alpha}<(-2 \log \alpha)^{1 / 2}+4.5 \times 10^{-4} \text { for } 0<\alpha<1 / 2
$$

it can be verified that the right side of $(21)$ is less than $1-\alpha$ for $0<\alpha<1 / 2$.

Table 1 gives values of the upper bound in (21) for some common values of $\alpha$.

As in section 3.1, it can be shown that $J_{n}$ is locally uniformly robust over $\mathscr{S}_{0}$ but not $\mathscr{G}_{1}$.

\section{Conclusion}

Although the main emphasis of this paper is on the asymptotic behaviour of the $t$-test and binomial confidence intervals, the problems they illustrate are typical of many statistical 
Table 1. Some values of the bound in (21)

\begin{tabular}{ll}
\hline $1-\alpha$ & $1-\exp \left(-4^{-1} z_{\alpha / 2}^{2}\right)$ \\
\hline 0.70 & 0.24 \\
0.80 & 0.34 \\
0.90 & 0.49 \\
0.95 & 0.62 \\
0.98 & 0.74 \\
0.99 & 0.81 \\
\hline
\end{tabular}

procedures derived through delta method arguments. That is, such procedures are usually pointwise robust but not uniformly robust.

To illustrate this somewhat more broadly, suppose that $\mathbf{X}_{i}=\left(X_{i 1}, \ldots, X_{i p}\right), i=1, \ldots, n$ are $n$ i.i.d. vectors and let $T_{i}=h\left(X_{i}\right)$ with $\eta=E\left(T_{i}\right)$ and $\tau^{2}=\operatorname{Var}\left(T_{i}\right)<\infty$, both independent of $i$. Then tests or confidence intervals for $\eta$ can be based on $n^{1 / 2}(\bar{T}-\eta) \hat{\tau}^{-1}$ where $\hat{\tau}$ is a consistent estimator of $\tau$, and it follows from the results for the $t$-test that the procedures are pointwise but not uniformly robust. The following are two applications.

1. Tests or confidence intervals for the variance of i.i.d. variables $X_{i}$, based on

$$
n^{-1 / 2} \hat{\tau}^{-1} \sum\left(X_{i}-\bar{X}\right)^{2}=n^{1 / 2} \hat{\tau}^{-1}\left(\sum X_{i}^{2} / n\right)-n^{-1 / 2} \hat{\tau}^{-1}\left(n^{1 / 2} \bar{X}\right)^{2}
$$

Since the left side is independent of $\xi=E\left(X_{i}\right)$, suppose without loss of generality that $\xi=0$. Then the second term on the right side tends to 0 in probability and the robustness results of the preceding paragraph apply with $p=1$ and $T_{i}=X_{i}^{2}$.

2. Tests or confidence intervals for the correlation coefficient of a bivariate distribution based on

$$
\frac{\Sigma\left(X_{i 1}-X_{\cdot 1}\right)\left(X_{i 2}-X_{\cdot 2}\right)}{n^{1 / 2} \hat{\tau}}=\frac{n^{1 / 2}\left(\sum X_{i 1} X_{i 2} / n\right.}{\hat{\tau}}-\frac{\left(n^{1 / 2} X_{\cdot 1}\right)\left(n^{1 / 2} X_{\cdot 2}\right)}{n^{1 / 2} \hat{\tau}}
$$

with $p=2, T_{i}=X_{i 1} X_{i 2}$ and $X_{. j}=n^{-1} \Sigma_{i} X_{i j}$.

The corresponding binomial result also holds much more generally. Let $X_{1}, \ldots, X_{n}$ be $n$ i.i.d. random variables whose possible values are the integers $\geqslant a$ and whose distribution is a one-parameter exponential family

$$
\operatorname{Pr}_{\theta}(X=x)=\exp \{\eta(\theta) x-A(\theta)\} h(x) ; \quad x=a, a+1, \ldots ; \theta \in\left(\theta_{0}, \theta_{1}\right) .
$$

Suppose that

$$
\operatorname{Pr}_{\theta}(X=a) \rightarrow 1 \quad \text { as } \quad \theta \rightarrow \theta_{0}
$$

and let $\xi=E_{\theta}(X)$. Then $\xi \rightarrow a$ as $\theta \rightarrow \theta_{0}$. Let $\tau^{2}=\operatorname{Var}_{\theta}(\bar{X})=n^{-1} \operatorname{Var}_{\theta}(X)=n^{-1} g(\xi)$, say, where $g$ is a continuous function. Equation (22) implies that $g(a)=0$. Let $\hat{\tau}^{2}=n^{-1} g(\bar{X})$. The confidence interval for $\xi$

$$
I_{n}=\bar{X} \pm z_{a} \sqrt{g(\bar{X}) / n}
$$

is pointwise but not uniformly robust because $\operatorname{Pr}_{\theta}\left(\xi \in I_{n}\right) \rightarrow 0$ as $\theta \rightarrow \theta_{0}$. The Poisson, truncated Poisson, and negative binomial are included in this situation.

Distribution-free tests such as the Wilcoxon and other rank tests are somewhat safer in this 
respect because of their (trivially achieved) uniform robustness. However, these distributionfree tests are not without problems of their own. For example, the size of the two-sample Wilcoxon test is not even pointwise robust if the population distributions are not identical, whereas the two-sample $t$-test is, as long as the population means are the same.

Extensions of the present results to the $k$-sample $F$ test are treated in Chen \& Loh (1990) and a more detailed analysis of the binomial confidence interval problem is given in Chen (1990).

\section{Acknowledgements}

We thank T. Severini for help in the proof of equation (12) and an associate editor for many helpful suggestions.

The first author's research was supported by National Science Foundation grant DMS 84-01388. The second author's research was supported by National Science Foundation grants DMS 85-02303 and DMS 88-03271, Air Force Office of Scientific Research grant ISSA 86-0068, and an IBM Junior Faculty Research Fellowship at the T. J. Watson Research Centre.

\section{References}

Abramowitz, M. \& Stegun, I. A. (1965). Handbook of mathematical functions. Dover, New York.

Bahadur, R. R. \& Savage, L. J. (1956). The nonexistence of certain statistical procedures in nonparametric problems. Ann. Math. Statist. 27, 1115-1122.

Benjamini, Y. (1983). Is the $t$-test really conservative when the parent distribution is long-tailed? J. Amer. Statist. Assoc. 78, 645-654.

Blyth, C. R. \& Still, H. A. (1983). Binomial confidence intervals. J. Amer. Statist. Assoc. 78, 108-116.

Chen, H. (1990). The accuracy of approximate intervals for a binomial parameter. J. Amer. Statist. Assoc. 85, 514-518.

Chen, H. \& Loh, W.-Y. (1990). Uniform robustness against nonnormality of the $t$ and $F$ tests. To appear. Chung, K. L. (1946). The approximate distribution of Student's statistic. Ann. Math. Statist. 17, 447-465. Cressie, N. (1980). Relaxing assumptions in the one-sample $t$-test. Austr. J. Statist. 22, 143-153.

Feller, W. (1966). An introduction to probability theory and its applications. Volume II. Wiley, New York.

Gleser, L. J. \& Hwang, J. T. (1987). The nonexistence of $100(1-\alpha) \%$ confidence sets of finite expected diameter in errors-in-variables and related models. Ann. Statist. 15, 1351-1362.

Herrendoerfer, G. \& Feige, K. D. (1985). A combinatorial method in robustness research and two applications. In Robustness of statistical methods and nonparametric statistics (eds. D. Rasch \& M. L. Tiku), 53-57, Reidel Publ. Co, Holland.

Herrendoerfer, G., Rasch, D. \& Feige, K. D. (1983). Robustness of statistical methods II. Methods for the one-sample problem. Biometrical J. 4, 327-343.

Ibragimov, I. A. (1956). On the composition of unimodal distributions. Theor. Probab. Appl. 1, 255-260.

Johnson, N. L. \& Kotz, S. (1969). Distributions in statistics-discrete distributions. Wiley, New York.

Lehmann, E. L. (1975). Nonparametrics: statistical methods based on ranks. Holden Day, San Francisco. Lehmann, E. L. (1986). Testing statistical hypotheses. Second edition. Wiley, New York.

Loh, W.-Y. (1984). Bounds on AREs for restrictred classes of distributions defined via tail-orderings. Ann. Statist. 12, 685-701.

Miller, R. G., Jr. (1986). Beyond ANOVA, basics of applied statistics. Wiley, New York.

Prakasa Rao, B. L. S. (1987). Asymptotic theory of statistical inference. Wiley, New York.

van Zwet, W. R. (1964). Convex transformations of random variables. Math. Centrum, Amsterdam.

Received July 1988, in final form October 1989

E. L. Lehmann, Department of Statistics, University of California, Berkeley, CA 94720. 